\title{
Boston type I keratoprosthesis-donor cornea interface evaluated by high-definition spectral- domain anterior segment optical coherence tomography
}

\author{
This article was published in the following Dove Press journal: \\ Clinical Ophthalmology \\ 24 August 2012 \\ Number of times this article has been viewed
}

\section{Ana G Alzaga Fernandez* \\ Nathan M Radcliffe* \\ Kimberly C Sippel \\ Mark I Rosenblatt \\ Priyanka Sood \\ Christopher E Starr \\ Jessica B Ciralsky \\ Donald J D'Amico \\ Szilárd Kiss}

Department of Ophthalmology, Weill Cornell Medical College, New YorkPresbyterian Hospital, New York, NY, USA

*These authors contributed equally to this work and both are considered principal authors
Correspondence: Szilárd Kiss Weill Cornell Medical College, 1305 York Ave, New York, NY 1002I, USA

Tel +l 6469622020

Fax + I 6469620604

Emailszk700I@med.cornell.edu
Background: The purpose of this study was to assess whether the resolution offered by two different, recently commercially available high-resolution, spectral-domain anterior segment optical coherence tomography (AS-OCT) instruments allows for detailed anatomic characterization of the critical device-donor cornea interface in eyes implanted with the Boston type I permanent keratoprosthesis.

Methods: Eighteen eyes of 17 patients implanted with the Boston type I keratoprosthesis were included in this retrospective case series. All eyes were quantitatively evaluated using the Cirrus HD-OCT while a subset (five eyes) was also qualitatively imaged using the Spectralis Anterior Segment Module. Images from these instruments were analyzed for evidence of epithelial migration onto the anterior surface of the keratoprosthesis front plate, and presence of a vertical gap between the posterior surface of the front plate and the underlying carrier donor corneal tissue. Quantitative data was obtained utilizing the caliper function on the Cirrus HD-OCT.

Results: The mean duration between AS-OCT imaging and keratoprosthesis placement was 29 months. As assessed by the Cirrus HD-OCT, $83 \%$ of eyes exhibited epithelial migration over the edge of the front plate. Fifty-six percent of the keratoprosthesis devices displayed good apposition of the device with the carrier corneal donor tissue. When a vertical gap was present (44\% of eyes), the mean gap was 40 (range 8-104) microns. The Spectralis Anterior Segment Module also displayed sufficient resolution to allow for similar characterization of the device-donor cornea interface.

Conclusion: Spectral-domain AS-OCT permits high resolution imaging of the keratoprosthesis device-donor cornea interface. Both the Cirrus HD-OCT and the Spectralis Anterior Segment module allowed for visualization of epithelial coverage of the device-donor cornea interface, as well as identification of physical gaps. These imaging modalities, by yielding information in regard to integration of the keratoprosthesis with surrounding corneal tissue, may help identify those at risk for keratoprosthesis-related complications, such as extrusion and endophthalmitis, and hence guide clinical management.

Keywords: keratoprosthesis, anterior segment optical coherence tomography, epithelialization

\section{Introduction}

Improved outcomes have resulted in increased use of permanent keratoprostheses for visual rehabilitation. The Boston type I keratoprosthesis is one of the most widely used corneal prostheses in the United States and abroad. ${ }^{1-3}$ It consists of a donor corneal button, serving as a carrier, sandwiched between a plastic polymethyl methacrylate front 
plate and a polymethyl methacrylate or titanium back plate. ${ }^{4,5}$ The stem connecting the front plate with the back plate provides an optically clear channel for vision. The carrier donor corneal button is sutured into place in standard penetrating keratoplasty fashion. A bandage contact lens is often placed over the device and the ocular surface to minimize the risk of desiccation around the stem.

Since its development in the 1960s and approval by the US Food and Drug Administration in 1992, the Boston keratoprosthesis has undergone a number of significant design changes. The medical management of keratoprosthesis patients, which now includes long-term use of topical vancomycin, has also undergone significant changes. These advances have resulted in marked improvement in retention rates and visual outcomes. ${ }^{6-9}$ However, despite these innovations, use of the Boston keratoprosthesis is still associated with complications such as tissue necrosis as well as infection. ${ }^{10-21}$ The risk of many complications relates directly to the extent to which the device is integrated with the surrounding corneal tissue. Specifically, in the case of the Boston type I keratoprosthesis, the potential space between the front plate/ stem and carrier corneal donor tissue is believed to be linked to the ease with which micro-organisms and proteolytic enzymes can gain access to the corneal stroma or to the anterior chamber, and hence to the risk of endophthalmitis or tissue necrosis around the stem.

Two recent case reports have described epithelial tissue, instead of stopping at the edge of the front plate, extending over the edge of the front plate and for a variable distance onto the anterior surface of the front plate in eyes implanted with more recent models of the Boston type I keratoprosthesis. ${ }^{22,23}$ The tissue was confirmed to be epithelium by light microscopic and immunohistochemical analysis. This epithelium, by spanning the junction between the front plate/stem of the keratoprosthesis and the carrier donor corneal tissue, presumably confers a beneficial effect by constituting a barrier to micro-organisms and proteolytic enzymes.

Recent studies have examined the utility of imaging modalities such as anterior segment optical coherence tomography (AS-OCT) in providing additional information in regard to an implanted keratoprosthesis and its relationship to the eye. AS-OCT offers cross-sectional views of the device in situ without necessitating direct contact with the eye. ${ }^{24,25}$ Newer-generation spectral-domain AS-OCT instrumentation provides increasingly high resolution imaging capability.

The goal of the present study was to evaluate the anatomic characteristics of the critical device-donor cornea interface in eyes implanted with the Boston type I keratoprosthesis using two different, recently commercially available high-resolution, spectral-domain AS-OCT imaging instruments. The focus was specifically on the capability of the instruments to identify and further characterize epithelial migration onto the front plate, and physical gaps between the front plate/stem (specifically, the posterior surface of the front plate) and the adjacent carrier donor corneal tissue.

\section{Materials and methods}

The patients included in this retrospective case series were derived from the Cornea Service of the Weill Cornell Medical College Department of Ophthalmology. Eighteen eyes implanted with the threadless model of the type I Boston permanent keratoprosthesis were studied. Demographic and clinical data for each patient, including indication for keratoprosthesis, were recorded. All eyes were quantitatively evaluated using the Cirrus HD-OCT (Carl Zeiss Meditec Inc, Dublin, CA). A subset (five eyes) was additionally qualitatively imaged using the Spectralis Anterior Segment Module (Heidelberg Engineering, Heidelberg, Germany).

Patients underwent imaging in four quadrants with the Cirrus HD-OCT (superior, inferior, nasal, temporal) using a 5-line high-resolution raster scan. The emphasis during image acquisition was placed on visualization of the device-donor cornea interface. Quantitative data were obtained using the caliper function on the Cirrus HD-OCT. In those patients who underwent imaging with the Spectralis Anterior Segment Module, line scans of $3 \mathrm{~mm}$ in length with $0.25 \mathrm{~mm}$ spacing were utilized.

The Weill Cornell Medical College and New York/ Presbyterian Hospital institutional review board approved this study according to local and national guidelines. Health Insurance Portability and Accountability Act compliance was maintained. Care of the patients in this study was in accordance with the Declaration of Helsinki and all federal and state laws.

\section{Results}

All 18 eyes underwent implantation with the Boston type I keratoprosthesis between 2007 and 2012. AS-OCT imaging was obtained at a single point between one month and 5 years after implantation. The mean single time point of acquisition was 29 months after implantation. The most frequent indication for keratoprosthesis placement was previous failed corneal transplantation initially performed secondary to pseudophakic bullous keratopathy (five of 18 eyes) 
or trauma (three of 18 eyes). Seventeen of the 18 eyes had a keratoprosthesis designed for aphakia placed (94\%); only one had a pseudophakic design. Seventeen of the 18 eyes underwent implantation with a keratoprosthesis with a polymethyl methacrylate backplate and only one with a titanium back plate. Eleven of the 18 eyes had a Kontur ${ }^{\mathrm{TM}}$ bandage contact lens (Kontur Kontact Lens Co, Inc, Hercules, CA) in place at the time of AS-OCT imaging; in these patients, the bandage contact lens had been in place for at least one month prior to imaging.

Epithelium was found to extend onto the anterior surface of the front plate in at least one image quadrant in 15 of the 18 eyes $(83 \%)$ imaged by Cirrus HD-OCT, confirming findings derived from slit-lamp examination (Figure 1). This epithelium was visualized by AS-OCT as a highly reflective layer extending from the donor cornea over the edge of the front plate (Figure 1B). The epithelial tissue could also be visualized using the Spectralis Anterior Segment Module (Figure 1C). One patient was imaged using the Cirrus HDOCT before and after the epithelium was pushed back using a Merocel ${ }^{\mathrm{TM}}$ sponge (Medtronic, Jacksonville, FL, Figure 2). Of the 15 eyes exhibiting epithelium spanning the devicedonor cornea interface by Cirrus HD-OCT imaging, eight had a Kontur bandage contact lens in place, and seven did not.

The extent of growth of this epithelium onto the front plate could more readily be measured in eyes without a Kontur bandage contact lens in place at the time of AS-OCT acquisition because the tear meniscus and debris
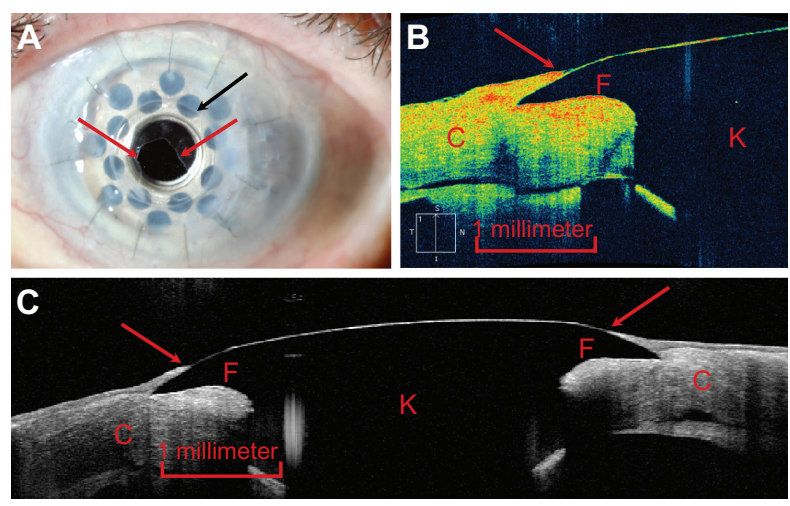

Figure I (A) Slit-lamp photograph demonstrating epithelium migrating onto the anterior surface of the front plate of the Boston type I keratoprosthesis (red arrows). ${ }^{23}$ The edge of the front plate is identified by a black arrow. (B) Zeiss Cirrus HD-OCT image of the junction between the keratoprosthesis device (identified by the letter "K") and the carrier donor corneal tissue ("C") offering a cross-sectional view of the device-donor cornea interface. The epithelial tissue (red arrow) is visualized and seen to extend onto the front plate ("F") and hence to cover the gap between the device and the carrier donor corneal tissue. (C) Heidelberg Spectralis Anterior Segment Module image of the device offering a comparable cross-sectional view, encompassing the entire front plate. Epithelial tissue coverage of the devicedonor cornea interface (red arrows) is again visualized. under the contact lens made identification of the leading edge of the epithelium more difficult. In these seven eyes without a bandage contact lens, the extent of growth as measured utilizing the Cirrus HD-OCT ranged from 216 to 2272 microns, with a mean of 1045 microns.

Ten of the 18 eyes (56\%) imaged by Cirrus HD-OCT exhibited excellent apposition of the posterior surface of the keratoprosthesis front plate and the carrier donor corneal tissue. In the remaining eight eyes (44\%), a measurable space between the posterior surface of the front plate and the underlying carrier corneal tissue was evident in at least one image quadrant (Figure 3A). This gap measured on average 40 microns in the vertical dimension with a range of 8-104 microns. Of the eight eyes exhibiting a vertical gap by Cirrus HD-OCT imaging, six had a Kontur bandage contact lens in place, and two did not. Notably, the three patients in whom no epithelium spanning the device-donor cornea interface could be identified by Cirrus HD-OCT, all exhibited a vertical gap. The Spectralis Anterior Segment Module similarly allowed for identification of a vertical gap (Figure 3B).

\section{Discussion}

Two studies have examined the utility of AS-OCT in providing information in regard to an implanted Boston type I keratoprosthesis and its relationship to the eye. ${ }^{24,27}$ In these studies, the AC Cornea OCT prototype (OTI, Quebec, Canada) was utilized. The stability of the keratoprosthesisdonor cornea interface was specifically examined by Garcia et al. ${ }^{25}$ Physical gaps between the posterior surface of the front plate and the carrier donor corneal tissue were visualized, albeit not examined in a quantitative fashion. No change in the keratoprosthesis-donor cornea interface (ie, no increased gaping or fluid escape) was noted with dynamic AS-OCT imaging in all patients when pressure was placed on the eye with a cotton-tip applicator.

To the best of our knowledge, this current report is the first to utilize commercially available high-resolution, spectraldomain AS-OCT imaging instruments for visualization of an implanted Boston type I keratoprosthesis. Both the Zeiss Cirrus HD-OCT and the Heidelberg Spectralis Anterior Segment Module offered detailed information in regard to the device-donor cornea interface, with both instruments allowing for imaging of epithelial migration onto the anterior surface of the front plate, as well as physical gaps between the front plate/stem and the carrier donor corneal tissue. Further quantitative information in regard to these findings could be obtained utilizing the caliper function on the Cirrus HD-OCT. The Spectralis Anterior Segment Module also has potential 

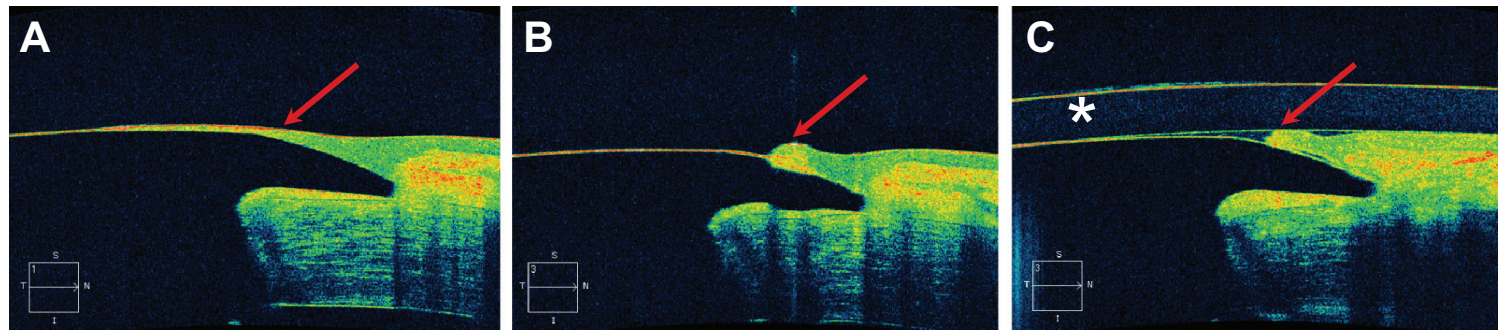

Figure 2 Cirrus HD-OCT images of the interface between the keratoprosthesis device and the carrier donor corneal tissue. (A) Epithelial tissue is visualized growing onto the anterior surface of the front plate (red arrow). (B) In the same patient, the epithelium was pushed back with a Merocel ${ }^{\mathrm{TM}}$ sponge at the slit-lamp and now appears as a scroll (red arrow). (C) The same patient after placement of a Kontur ${ }^{\mathrm{TM}}$ bandage contact lens (identified by an asterisk).

caliper functionality; however, this was not available on the instrument used in this study.

Epithelial coverage of the junction between the keratoprosthesis front plate and the carrier donor corneal tissue was identified by high-resolution AS-OCT in the majority of patients. The actual extent of epithelial migration could be most readily measured in patients in whom a bandage contact lens was not present because the leading edge of the epithelium could be readily identified. In only one patient did this epithelial coverage, the presence of which in general is considered beneficial, extend $2 \mathrm{~mm}$ onto the front plate, which was sufficient to encroach on the visual axis. The epithelium can be readily scrolled back at the slit-lamp using a Merocel sponge, as was captured by Cirrus HD-OCT in one patient (Figure 2). An association between the presence of epithelial tissue and coverage by a bandage contact lens was not apparent.

In contrast with evaluation of the presence of epithelium, the degree of apposition of the posterior surface of the front plate with the carrier donor corneal tissue does not lend itself readily to slit-lamp examination. A measurable vertical gap of any degree was identified in close to half of the patients by AS-OCT imaging. However, some of these vertical gaps were quite small (down to eight microns) and determination of the degree of vertical gap associated with clinical consequences will require further investigation. Interestingly, the three eyes without evidence of epithelial coverage of the edge of the front plate also demonstrated a vertical gap, although this study does not allow for conclusions in regard to causality.

This study indicates that high-resolution, spectral-domain AS-OCT offers detailed information in regard to the critical device-donor cornea interface that complements and expands the information that can be obtained by slit-lamp examination. Many of these patients require lifelong interventions such as prophylactic topical antibiotics ${ }^{12}$ and the wearing of a bandage contact lens in order to keep complications such as infection, desiccation, and melting around the stem at bay. ${ }^{26,27}$ The risk of many of these complications is linked to the integrity of the device-donor cornea interface. By providing information in regard to the degree of integration of the device, ie, extension of epithelium over the edge of the front and lack of physical gaps, these commercially available AS-OCT imaging modalities may play a role in determining optimal clinical management of patients with a Boston type I keratoprosthesis.
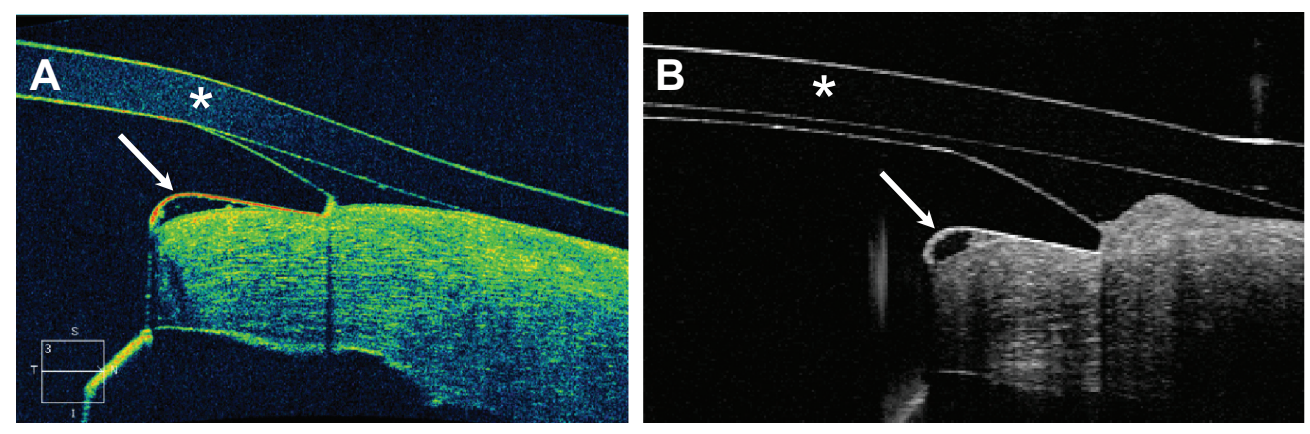

Figure 3 Imaging of the device-donor cornea interface with (A) the Cirrus HD-OCT and (B) the Spectralis Anterior Segment Module.

Notes: A vertical gap between the posterior surface of the front plate of the keratoprosthesis and the carrier donor corneal tissue can be visualized with both imaging modalities (white arrows). A Kontur ${ }^{\mathrm{TM}}$ bandage contact lens (asterisk) is seen in both images. 


\section{Acknowledgment}

This research was supported in part by an unrestricted departmental grant from the Research to Prevent Blindness.

\section{Disclosure}

NR serves as a consultant for Carl Zeiss Meditec Inc. None of the authors hold any proprietary interest in the material presented.

\section{References}

1. Ament JD, Todani A, Pineda R, et al. Global corneal blindness and the Boston keratoprosthesis type 1. Am J Ophthalmol. 2010;149(4): 537-539.

2. Colby KA, Koo EB. Expanding indications for the Boston keratoprosthesis. Curr Opin Ophthalmol. 2011;22(4):267-273.

3. Aldave AJ, Sangwan VS, Basu S, et al. International results with the Boston type I keratoprosthesis. Ophthalmology. April 17, 2012. [Epub ahead of print.]

4. Traish AS, Chodosh J. Expanding application of the Boston type 1 keratoprosthesis due to advances in design and improved postoperative therapeutic strategies. Semin Ophthalmol. 2010;25(5-6):239-243.

5. Doane MG, Dohlman CH, Bearse G. Fabrication of a keratoprosthesis. Cornea. 1996;15(2):179-184.

6. Aquavella JV, Qian Y, McCormick GJ, Palakuru JR. Keratoprosthesis: the Dohlman-Doane device. Am J Ophthalmol. 2005;140(6):1032-1038.

7. Harissi-Dagher M, Khan BF, Schaumberg DA, Dohlman CH. Importance of nutrition to corneal grafts when used as a carrier of the Boston keratoprosthesis. Cornea. 2007;26(5):564-568.

8. Klufas MA, Colby KA. The Boston keratoprosthesis. Int Ophthalmol Clin. 2010;50(3):161-175.

9. Khan BF, Harissi-Dagher M, Khan DM, Dohlman CH. Advances in Boston keratoprosthesis: enhancing retention and prevention of infection and inflammation. Int Ophthalmol Clin. 2007;47(2):61-71.

10. Nouri M, Terada H, Alfonso EC, Foster CS, Durand ML, Dohlman CH. Endophthalmitis after keratoprosthesis: incidence, bacterial causes, and risk factors. Arch Ophthalmol. 2001;119(4):484- 489.

11. Ayres BD. Characteristics of endophthalmitis in patients with the Boston keratoprosthesis. Cornea. 2012;31(7):846.

12. Durand ML, Dohlman CH. Successful prevention of bacterial endophthalmitis in eye with Boston keratoprosthesis. Cornea. 2009;28(8): 896-901.

13. Chew H, Ayres BD, Hammersmith KM, et al. Boston keratoprosthesis outcomes and complications. Cornea. 2009;28(9):989-996.
14. Zerbe BL, Belin MW, Ciolino JB. Results from the multicenter Boston type 1 keratoprosthesis study. Ophthalmology. 2006;113(10): 1779-1784.

15. Jain V, Mhatre K, Shome D, Pineda R. Fungal keratitis with the type 1 Boston keratoprosthesis: early Indian experience. Cornea. 2012;31(7): 841-843.

16. Robert MC, Moussally K, Harissi-Dagher M. Review of endophthalmitis following Boston keratoprosthesis type 1. Br J Ophthalmol. 2012; 96(6):776-780.

17. Chan CC, Holland EJ. Infectious endophthalmitis after Boston type 1 keratoprosthesis implantation. Cornea. 2012;31(4):346-349.

18. Ramchandran RS, Diloreto DA Jr, Chung MM, et al. Infectious endophthamitis in adult eyes receiving Boston type 1 keratoprosthesis. Ophthalmology. 2012;119(4):674-681.

19. Yu JF, Huang YF. Characteristics of endophthalmitis with Boston keratoprosthesis. Cornea. 2012;31(5):594.

20. Greiner MA, Li JY, Mannis MJ. Longer-term vision outcomes and complications with the Boston type 1 keratoprosthesis at the University of California, Davis. Ophthalmology. 2011;118(8):1543-1550.

21. Utine CA, Tzu JH, Akpek EK. Clinical features and prognosis of Boston type 1 keratoprosthesis-associated corneal melt. Ocul Immunol Inflamm. 2011;19(6):413-418.

22. Kiang L, Rosenblatt MI, Sartaj R, et al. Surface epithelium of the type I Boston keratoprosthesis front plate: immunohistochemical and high-definition optical coherence tomography characterization. Graefes Arch Clin Exp Ophthalmol. 2012 Aug;250(8):1195-9.

23. Khalifa YM, Davis D, Mamalis N, Moshirfar M. Epithelial growth over the optic surface of the type 1 Boston keratoprosthesis: histopathology and implications for biointegration. Clin Ophthalmol. 2010;4: 1069-1071.

24. Garcia JP Jr, De la Cruz J, Rosen RB, Buxton DF. Imaging implanted keratoprosthesis with anterior-segment optical coherence tomography and ultrasound biomicroscopy. Cornea. 2008;27(2):180-188.

25. Garcia JP Jr, Ritterband DC, Buxton DF, De la Cruz J. Evaluation of the stability of Boston Type I keratoprosthesis-donor cornea interface using anterior segment optical coherence tomography. Cornea. 2010;29(9): 1031-1035.

26. Dohlman CH, Dudenhoefer EJ, Khan BF, Morneault S. Protection of the ocular surface after keratoprosthesis surgery: the role of soft contact lenses. CLAO J. 2002;28(2):72-74.

27. Harissi-Dagher M, Beyer J, Dohlman CH. The role of soft contact lenses as an adjunct to the Boston keratoprosthesis. Int Ophthalmol Clin. 2008;48(2):43-51.
Clinical Ophthalmology

\section{Publish your work in this journal}

Clinical Ophthalmology is an international, peer-reviewed journal covering all subspecialties within ophthalmology. Key topics include: Optometry; Visual science; Pharmacology and drug therapy in eye diseases; Basic Sciences; Primary and Secondary eye care; Patient Safety and Quality of Care Improvements. This journal is indexed on Submit your manuscript here: http://www.dovepress.com/clinical-ophthalmology-journal

\section{Dovepress}

PubMed Central and CAS, and is the official journal of The Society of Clinical Ophthalmology (SCO). The manuscript management system is completely online and includes a very quick and fair peer-review system, which is all easy to use. Visit http://www.dovepress.com/ testimonials.php to read real quotes from published authors. 